\title{
Why macroscopic quantum tunnelling in Josephson junctions differs from tunnelling of a quantum particle
}

\author{
A. O. Sboychakov ${ }^{1,2}$, Sergey Savel'Ev ${ }^{1,3}$, A. L. Rakhmanov ${ }^{1,2,3}$ and Franco Nori ${ }^{1,4}$ \\ ${ }^{1}$ Frontier Research System, The Institute of Physical and Chemical Research (RIKEN) - Wako-shi, \\ Saitama, 351-0198, Japan \\ ${ }^{2}$ Institute for Theoretical and Applied Electrodynamics Russian Academy of Sciences - 125412 Moscow, Russia \\ ${ }^{3}$ Department of Physics, Loughborough University - Loughborough LE11 3TU, UK \\ ${ }^{4}$ MCTP, CSCS, Department of Physics, University of Michigan - Ann Arbor, MI 48109-1040, USA
}

received 14 June 2007; accepted in final form 17 August 2007

published online 17 September 2007

PACS 74.50.+r - Tunneling phenomena; point contacts, weak links, Josephson effects

\begin{abstract}
We show that the macroscopic quantum tunnelling of a fluxon in a Josephson junction cannot be described, even qualitatively, as the tunnelling of a quantum particle in a potential $U(\varphi)$, where the phase difference $\varphi$ plays the role of particle position, if the length of the junction $d$ exceeds a fluxon length. We calculate the probability per unit time of tunnelling (or escape rate), $\Gamma$, which has a form $\Gamma=A \exp (-B)$. In contrast to particles, where the $B$ is proportional to $d$, our field-theory predicts a different behavior of $B$ for either usual, $0-\pi$, or stacks of Josephson junctions, giving rise to a renormalization of $\Gamma$ by many orders of magnitude.
\end{abstract}

Copyright (C) EPLA, 2007

Macroscopic quantum tunnelling (MQT) is one of the few manifestations of quantum effects in macroscopic systems [1]. MQT was experimentally observed [1] in Josephson junctions (JJs) in 1980s and has been studied for different Josephson systems. General interest on MQT is now fuelled not only by its fundamental interest but as a readout mechanism for phase qubits [2]. A new surge of interest on MQT occurred after the recent discovery of MQT in high-temperature layered superconductors $[3,4]$. The observed giant enhancement of MQT could be attributed to the spatial structure of the tunnelling fluxon [5]. This is in contrast to the standard approach, where MQT is associated with a quantum particle tunnelling through an effective potential barrier. The latter approach is correct only for very short junctions, when one can ignore the spatial dependence of the phase difference $\varphi$ across the junction. A naive guess would be that the spatial dependence of $\varphi$ should suppress the MQT because of an increase of the potential energy $U(\varphi)$ from $(\nabla \varphi)^{2}$. This is in analogy to the positive energy of a domain wall in a ferromagnet. However, further analysis shows that the total potential energy can decrease similarly to the decrease of energy for a ferromagnet having several domains.

Our work was motivated by the MQT recently observed in high-temperature layered superconductors. However, we emphasize that the problem we are considering is far more general and is also applicable to MQT in long Josephson junctions (see, e.g., [6]). Moreover, MQT in long Josephson junctions is of relevance for the so-called phase qubits which might provide basic elements for future quantum information processing devices (see ref. [2] for a review). These phase qubits are current-biased JJs driven by microwaves, and exhibiting MQT. Controlling the motion of Josephson vortices is also of interest for the design of novel types of $\mathrm{THz}$ and ratchet devices [7].

Using a field-theory approach, here we show that the fluxon tunnels through the formation of single nucleus followed by a classical motion through the contact. For a long junction this results in a huge renormalization of the tunnelling escape rate $\Gamma$, with respect to the "particle" approximation. The tunnelling probability differs for the usual and $0-\pi$ junctions: for usual junctions, $\log \Gamma(d)$ is proportional to $d$ for $d \ll \lambda_{J}$ and shows a maximum at $d \sim \lambda_{J}$; while $\log \Gamma(d)$ for $0-\pi$ junctions increases slower with $d$ for $d \ll \lambda_{J}$ and does not exhibit a maximum within the studied range of parameters. We also demonstrate that the strong renormalization of $\Gamma$ for stacks of intrinsic JJs occurs even for very short junctions of about $1 \mu \mathrm{m}$, in agreement with very recent experiments [4]. This offers potentially useful flexibility when designing readouts for phase qubits [2]. 
General model. - Consider a scalar field $\varphi$ in a finite volume $V$ with surface $S$. The field action is given by $(c=1)$

$$
\begin{aligned}
& \mathcal{S}(t)=\int_{-\infty}^{t} \mathrm{~d} t\left[\mathcal{L}(t)+\varepsilon_{0} \int_{S} \mathrm{~d} S \varphi(t, \mathbf{x}) f(\mathbf{x})\right], \\
& \mathcal{L}(t)=\varepsilon_{0} \int_{V} \mathrm{~d} \mathbf{x}\left[\frac{1}{2}\left(\frac{\partial \varphi}{\partial t}\right)^{2}-\frac{1}{2}(\nabla \varphi)^{2}-U(\varphi)\right],
\end{aligned}
$$

where $\mathcal{L}$ is the Lagrangian and $\varepsilon_{0}$ is an energy scale. Hereafter we ignore dissipation, which was shown [8] to be negligible. The equality $\delta S / \delta \varphi(t, \mathbf{x})=0$ determines the classical equation of motion for the field $\varphi(t, \mathbf{x})$. The surface term in eq. (1) corresponds to the external force acting on the field. It leads to the boundary condition $\partial \varphi /\left.\partial n\right|_{\mathbf{x} \in S}=f(\mathbf{x})$. We assume that the equation $\delta S / \delta \varphi=0$ has at least two static solutions corresponding to two energy minima. One of them, $\varphi_{-}(\mathbf{x})$, corresponds to a global minimum, whereas the second one, $\varphi_{0}(\mathbf{x})$, is a local energy minimum. We calculate the probability $\Gamma$ (per unit time) of transition from the local minimum to the global one. In the semiclassical limit this probability can be written as [9] $\Gamma=\omega_{0} \sqrt{30 B / \pi} \exp (-B)$, where $\omega_{0}$ is the oscillation frequency of the field near $\varphi_{0}(\mathbf{x})$, $B=i \mathcal{S}_{\mathrm{cl}}(i \infty) / \hbar$, and $\mathcal{S}_{\mathrm{cl}}$ is the action eq. (1) calculated along classical trajectories. Introducing the imaginary time $t=i \tau$, it was obtained [9]

$$
B=-\frac{2}{\hbar} \int_{0}^{\infty} \mathrm{d} \tau\left[\mathcal{L}(\tau)+\varepsilon_{0} \int_{S} \mathrm{~d} S \varphi(\tau, \mathbf{x}) f(\mathbf{x})\right] .
$$

The field $\varphi(\tau, \mathbf{x})$ in eq. (2) satisfies the imaginarytime classical equation of motion $\left(\partial^{2} / \partial \tau^{2}+\nabla^{2}\right) \varphi-$ $\partial U(\varphi) / \partial \varphi=0$ with the initial conditions $\partial \varphi /\left.\partial \tau\right|_{\tau=0}=0$, $\varphi(\infty, \mathbf{x})=\varphi_{0}(\mathbf{x})$.

The quantum tunnelling of a macroscopic system occurs with an appreciable probability only if the barrier is low. Then, we can use a perturbation approach and seek a solution of the form $\varphi(\tau, \mathbf{x})=\varphi_{0}(\mathbf{x})+\psi(\tau, \mathbf{x})$, with $|\psi| \ll$ $\left|\varphi_{0}\right|$. Expanding the potential $U(\varphi)$ in a series of $\psi$ and integrating by parts, we obtain

$$
\begin{aligned}
B= & \frac{2 \varepsilon_{0}}{\hbar} \int_{0}^{\infty} \mathrm{d} \tau \int_{V} \mathrm{~d} \mathbf{x}\left[\frac{1}{2} \psi\left(\hat{L}-\frac{\partial^{2}}{\partial \tau^{2}}\right) \psi\right. \\
& \left.+\frac{1}{6} \frac{\partial^{3} U\left(\varphi_{0}\right)}{\partial \varphi_{0}^{3}} \psi^{3}+\frac{1}{24} \frac{\partial^{4} U\left(\varphi_{0}\right)}{\partial \varphi_{0}^{4}} \psi^{4}+\ldots\right],
\end{aligned}
$$

where $\hat{L}=-\nabla^{2}+\partial^{2} U\left(\varphi_{0}\right) / \partial \varphi_{0}^{2}$. The perturbation $\psi(\tau, \mathbf{x})$ satisfies the equation of motion

$$
\left(\frac{\partial^{2}}{\partial \tau^{2}}-\hat{L}\right) \psi=\frac{1}{2} \frac{\partial^{3} U\left(\varphi_{0}\right)}{\partial \varphi_{0}^{3}} \psi^{2}+\frac{1}{6} \frac{\partial^{4} U\left(\varphi_{0}\right)}{\partial \varphi_{0}^{4}} \psi^{3}+\ldots,
$$

with zero boundary and initial conditions.

We consider the system of eigenfunctions $\psi_{n}$ of the operator $\hat{L}$ corresponding to the eigenvalues $\mu_{n}$

$$
\hat{L} \psi_{n}=\mu_{n} \psi_{n}, \quad \partial \psi_{n} /\left.\partial n\right|_{\mathbf{x} \in S}=0 .
$$

These functions are orthogonal and normalized $\int_{V} \mathrm{~d} \mathbf{x} \psi_{n} \psi_{m}=\delta_{n m}$. The solution $\psi$ can be expanded in the basis $\psi_{n}$ as $\psi(\tau, \mathbf{x})=\sum_{n} c_{n}(\tau) \psi_{n}(\mathbf{x})$. Multiplying eq. (4) by $\psi_{n}$ and performing space integration, we get the system of equations for $c_{n}(\tau)$

$$
\ddot{c}_{n}-\mu_{n} c_{n}=-\frac{1}{2} \sum_{m k} U_{n m k}^{(3)} c_{m} c_{k}-\frac{1}{6} \sum_{m k l} U_{n m k l}^{(4)} c_{m} c_{k} c_{l}
$$

with initial conditions $\dot{c}_{n}(0)=c_{n}(\infty)=0$. Here, dot means imaginary time derivative, and

$$
U_{n \ldots k}^{(i)}=-\int_{V} \mathrm{~d} \mathbf{x} \partial^{i} U\left(\varphi_{0}\right) / \partial \varphi_{0}^{i} \psi_{n} \ldots \psi_{k} .
$$

Substituting the series expansion for $\psi$ into eq. (3) and using eq. (6) we express $B$ in the form

$B=\frac{\varepsilon_{0}}{\hbar} \int_{0}^{\infty} \mathrm{d} \tau\left[\sum_{n m k} \frac{U_{n m k}^{(3)}}{6} c_{n} c_{m} c_{k}+\sum_{n m k l} \frac{U_{n m k l}^{(4)}}{12} c_{n} c_{m} c_{k} c_{l}+\ldots\right]$.

We enumerate the eigenvalues of $\hat{L}$ in ascending order $\left(\mu_{0}<\mu_{1}<\mu_{2}<\ldots\right)$. All $\mu_{n}$ 's are positive since $\varphi_{0}(\mathbf{x})$ corresponds to an energy minimum. With increasing external force $f(\mathbf{x})$, the state $\varphi_{0}(\mathbf{x})$ becomes unstable and $B=0$. At this point, several lowest $\mu_{n}$ 's are zero. At first, we assume that only the value $\mu_{0}$ vanishes. In this case, $\mu_{0} \ll \mu_{n}$ for $n>0$ when $f(\mathbf{x})$ is close to the critical value. According to eq. (8), the condition $B \rightarrow 0$ requires $c_{n} \rightarrow 0$. Thus, we can write a series expansion $c_{n}(\tau)=\mu_{0} c_{n}^{(0)}+\mu_{0}^{2} c_{n}^{(1)}+\ldots$ Substituting this expansion into eq. (6), we find that $c_{n}^{(0)}=0$ for $n>0$. In this case, the characteristic frequency $\omega_{0}$ in the expression for $\Gamma$ is $\omega_{0} \approx$ $\sqrt{\mu_{0}}$. We introduce new variables $c_{0}^{(0)}(\tau)=3 \alpha(\zeta) / U_{000}^{(3)}$, $\zeta=\omega_{0} \tau$. In the main approximation with respect to $\mu_{0}$, the system (6) with its initial conditions reduces to

$$
\frac{\mathrm{d}^{2} \alpha}{\mathrm{d} \zeta^{2}}-\alpha=-\frac{3}{2} \alpha^{2}, \quad \alpha(\infty)=\frac{\mathrm{d} \alpha(0)}{\mathrm{d} \zeta}=0 .
$$

After integration we get $\int_{\alpha(\zeta)}^{1} \mathrm{~d} \alpha /(\alpha \sqrt{1-\alpha})=\zeta$. Substituting $c_{0}^{(0)}$ into eq. (8) and performing time integration with the help of eqs. (9), we derive the expression for $B$ in the main approximation in $\mu_{0}$

$$
B^{(0)}=24 \varepsilon_{0} \mu_{0}^{5 / 2} /\left[5 \hbar\left(U_{000}^{(3)}\right)^{2}\right] .
$$

The equations for $c_{n}^{(1)}$ at $n>0$ follow from eqs. (6) and the series expansion of $c_{n}\left(\mu_{0}\right)$,

$$
\ddot{c}_{n}^{(1)}-\mu_{n} c_{n}^{(1)}=-\frac{1}{2} U_{n 00}^{(3)}\left(c_{0}^{(0)}\right)^{2} .
$$

The solution of this equation, with the appropriate initial conditions, can be written as $c_{n}^{(1)}(\tau)=$ $\left[9 U_{n 00}^{(3)} /\left(U_{000}^{(3)}\right)^{2}\right] \nu_{n}\left(\sqrt{\mu_{0}} \tau\right)$, where

$$
\begin{aligned}
\nu_{n}(\zeta)= & \frac{\mathrm{e}^{-\lambda_{n} \zeta}}{2 \mu_{0} \lambda_{n}} \int_{0}^{\infty} \mathrm{d} \zeta^{\prime} \alpha^{2}\left(\zeta^{\prime}\right) \mathrm{e}^{-\lambda_{n} \zeta^{\prime}} \\
& +\frac{1}{2 \mu_{0}} \int_{0}^{\zeta} \mathrm{d} \zeta^{\prime} \mathrm{e}^{\lambda_{n}\left(\zeta^{\prime}-\zeta\right)} \int_{0}^{\infty} \mathrm{d} \zeta^{\prime \prime} \alpha^{2}\left(\zeta^{\prime}+\zeta^{\prime \prime}\right) \mathrm{e}^{-\lambda_{n} \zeta^{\prime \prime}}
\end{aligned}
$$


and $\lambda_{n}=\sqrt{\mu_{n} / \mu_{0}} \gg 1$. Writing $c_{0}^{(1)}$ in the form $c_{0}^{(1)}(\tau)=$ $\left[9 /\left(U_{000}^{(3)}\right)^{2}\right] \gamma\left(\sqrt{\mu_{0}} \tau\right)$, we obtain, from eqs. $(6)$, for $\gamma(\zeta)$ :

$$
\frac{\mathrm{d}^{2} \gamma}{\mathrm{d} \zeta^{2}}-\gamma=-3 \alpha \gamma-3 \alpha \sum_{n \neq 0} v_{n}^{(3)} \nu_{n}-\frac{1}{2} v_{0}^{(4)} \alpha^{3},
$$

where $v_{n}^{(3)}=\left(U_{n 00}^{(3)}\right)^{2} / U_{000}^{(3)}, \quad v_{0}^{(4)}=U_{000}^{(4)} / U_{000}^{(3)}$. The initial conditions for eq. (13) are evidently $\gamma(\infty)=\mathrm{d} \gamma(0) / \mathrm{d} \zeta=0$. Substituting the obtained formulas into eq. (8) we derive the expression for the tunnelling exponent

$$
\begin{aligned}
B \cong & B^{(0)}\left\{1+\frac{45 \mu_{0}}{16 U_{000}^{(3)}} \int_{0}^{\infty} \mathrm{d} \zeta \alpha^{2}\right. \\
& \left.\times\left[3 \gamma+3 \sum_{n \neq 0} v_{n}^{(3)} \nu_{n}+\frac{1}{2} v_{0}^{(4)} \alpha^{2}\right]\right\} .
\end{aligned}
$$

To compute $B$, we should first solve the eigenvalue problem (5), and solve eqs. (9), (12), and (13). Similarly, higher-order corrections to $B$ could be obtained. The approach described above can be generalized for the case when several, $m$, eigenvalues are small at the critical external force. In this case, we should solve the system of $m$ nonlinear equations for $c_{n}^{(0)}(n \leqslant m)$, and then find corrections to $c_{n}$ using a perturbation approach. The existence of $m$ small eigenvalues indicates that there exists $m$ channels for tunnelling. The case of two small parameters $\mu_{0}$ and $\mu_{1}$ will be essential for the case considered below: the quantum tunnelling of a fluxon.

Tunnelling in Josephson junctions. - Here we apply our approach for the study of a fluxon tunnelling in a JJ. The geometry of the problem is shown in the inset of fig. 1. Two superconducting bars overlap a length $d$ in the $x$-direction. The length $l$ of the JJ in the $y$-direction is of the order of or larger than $d$. The current per unit $y$ length, $J$, flows through the junction in the $z$-direction. The properties of a JJ are described in terms of the gauge-invariant phase difference $\varphi$, which obeys the sine-Gordon equation. It can be shown that at $l \gg d$, the stationary solution $\varphi_{0}$, corresponding to an energy minimum, does not depend on $y$, and the $y$-dependence of $\psi_{n}$ contributes only to the higher-order corrections to $B$. In the geometry considered here we can write the sine-Gordon equation for $\varphi$ as

$$
\ddot{\varphi}-\varphi_{x x}+\sin \varphi=0,
$$

where the time $t$ and coordinate $x$ are normalized by the Josephson frequency $\omega_{J}$ and length $\lambda_{J}$. The current density $i(x)$ is given by the Josephson relation $i(x)=i_{0} \sin \varphi(x)$, where $i_{0}$ is the critical current density. We define the dimensionless current $j=J / d i_{0}$, potential $U(\varphi)=U_{0}-\cos \varphi$, and $\varepsilon_{0}=\Phi_{0} i_{0} \lambda_{J} l /\left(2 \pi c \omega_{J}\right)$, where $\Phi_{0}$ is the flux quantum. The boundary conditions for eq. (15) are $\mathrm{d} \varphi / \mathrm{d} x= \pm I / 2, x= \pm d / 2 \lambda_{J}$, where $I=j d / \lambda_{J}$.

The stationary solution $\varphi_{0}(x)$ of eq. (15) corresponding to an energy minimum can be written as $\int_{\tilde{\varphi}_{0}}^{\varphi_{0}(x)} \mathrm{d} \varphi / \sqrt{\cos \tilde{\varphi}_{0}-\cos \varphi}=\sqrt{2}|x|$, where the value

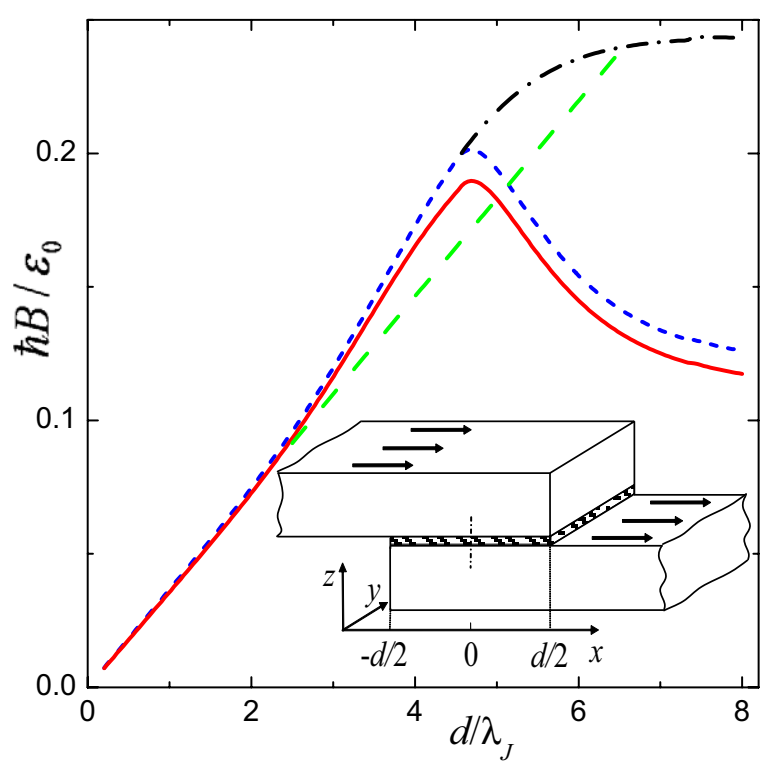

Fig. 1: (Color online) The logarithm $B$ of the escape rate $\Gamma$ vs. $d$ at $I / I_{c}(d)=0.99$ calculated in zeroth-order (dashed blue curve) and first-order (solid red curve) approximation. The dashed green line corresponds to eq. (16), i.e., the "particle" approximation. The schematics of the JJ is shown in the inset. The dash-dotted curve is calculated without taking into account the second small parameter.

$\tilde{\varphi}_{0}=\arccos \left[\cos \varphi_{0}\left(d / 2 \lambda_{J}\right)+I^{2} / 8\right]$ is found from the boundary condition. The solution of this equation exists if the current $I$ is less than the critical value $I_{c}(d)$. If $d \lesssim 4 \lambda_{J}$, the current density is approximately constant in the JJ and the function $I_{c}(d)$ increases linearly with $d$; if $d \gg \lambda_{J}$, the current flows near the junction edges and $I_{c}(d)$ reaches the saturation value $4 i_{0} \lambda_{J}$.

First, we compute $\varphi_{0}(x)$ and the set of eigenvalues and eigenfunctions of the operator $\hat{L}=-\mathrm{d}^{2} / \mathrm{d} x^{2}+\cos \varphi_{0}(x)$. Then, we numerically solve the system (6) by the method described above, and, finally, calculate $B$ in the main and first-order approximations. We find that the first-order correction to $B$ does not exceed a few percent for any $d$, if $I / I_{c}>0.97$. Thus, eq. (10) is applied for the calculation of $B$ with a good accuracy if the current $I$ is close to the critical value $I_{c}$. The dependence of $B$ on the junction length $d$ at $I / I_{c}(d)=0.99$ is shown in fig. 1 . At small $d$, the tunnelling exponent linearly increases with $d$, according to a well-known result obtained using an analogy with the quantum tunnelling of a particle $[4,10]$ :

$$
B_{\mathrm{QM}}=\frac{\Phi_{0} i_{0} l d}{2 \pi c \hbar \omega_{J}} \frac{24\left(1-j^{2}\right)^{5 / 4}}{5 j^{2}} .
$$

This formula follows directly from eq. (10). Naturally, if $d \ll \lambda_{J}$, we get $\varphi_{0}(x) \approx \arcsin (j)+j x^{2} / 2$. Neglecting the $x$-dependence of $\cos \varphi_{0}(x)$, from eq. (5) we obtain

$$
\mu_{0} \approx \sqrt{1-j^{2}}, \quad \psi_{0} \approx 1 / \sqrt{d}
$$

Substituting these values in eq. (10) we obtain eq. (16). 


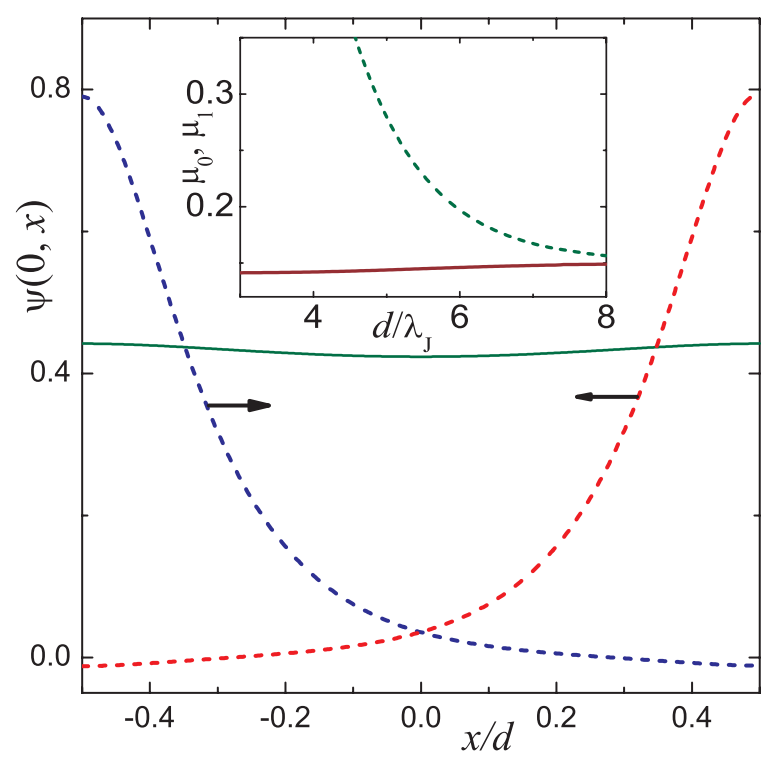

Fig. 2: (Color online) The shape $\psi(0, x)$ of the nucleus of a tunnelling fluxon at $d=2 \lambda_{J}$ (green solid curve) and $d=8 \lambda_{J}$ (red and blue dashed curves). At large $d$, two different functions $\psi(0, x)$ correspond to two channels of tunnelling: nucleation of a vortex (on the right sample edge) or an antivortex (on the left); $I / I_{c}(d)=0.99$. The dependence of $\mu_{0}$ (red solid curve) and $\mu_{1}$ (green dashed curve) on $d / \lambda_{J}$ is shown in the inset.

A pronounced departure of $B(d)$ from the linear law (16) arises at $d \gtrsim 4 \lambda_{J}$. The tunnelling exponent starts to decrease with the growth of $d$ and tends to a constant when $d \rightarrow \infty$. In this case, the eigenvalue $\mu_{1}$ becomes close to $\mu_{0}$ (see the inset in fig. 2) and one should take into account the existence of two tunnelling channels. The field $\psi$ can be written as $\psi(\tau, x)=c_{0}^{(0)}(\tau) \psi_{0}(x)+c_{1}^{(0)}(\tau) \psi_{1}(x)$. For small $d, c_{1}^{(0)}(\tau)=0$, whereas for $d \gg \lambda_{J}$ we have $\mu_{1} \approx \mu_{0}$ and $c_{1}^{(0)}(\tau) \approx \pm c_{0}^{(0)}(\tau)$. The function $\psi(0, x)$ is shown in fig. 2 for small and large $d$. At small $d, \psi(0, x) \approx$ const and the analogy between field and particle tunnelling is valid. For larger $d, \psi(0, x)$ is nonzero only near the right (vortex nucleation) or left (antivortex nucleation) edges of the junction, depending on the choice of the sign of $c_{1}^{(0)}(0)$. Thus, for $d \gg \lambda_{J}$ the MQT has two stages. First, the vortex (or antivortex) nucleus tunnels through the right (or left) edge of the junction. Then, this vortex (or antivortex) moves classically through the junction. The tunnelling probability starts to increase with the growth of $d$, due to the opening of the second tunnelling channel.

In addition to the usual JJ (0 junction), we calculate the probability of tunnelling for $0-\pi$ junctions. Such a junction naturally arises at the boundary of $d$ and $s$-wave superconductors [11] or can be fabricated using a ferromagnetic layer between $s$-wave superconductors. We consider the usual current-phase relation to be valid at $-d / 2<x<0$, whereas $i=i_{0} \sin (\phi+\pi)$ at $0<x<d / 2$. The ground state of a $0-\pi$ junction corresponds to a vortex with one-half of $\Phi_{0}$ confined at the boundary between " 0 " and " $\pi$ " regions.

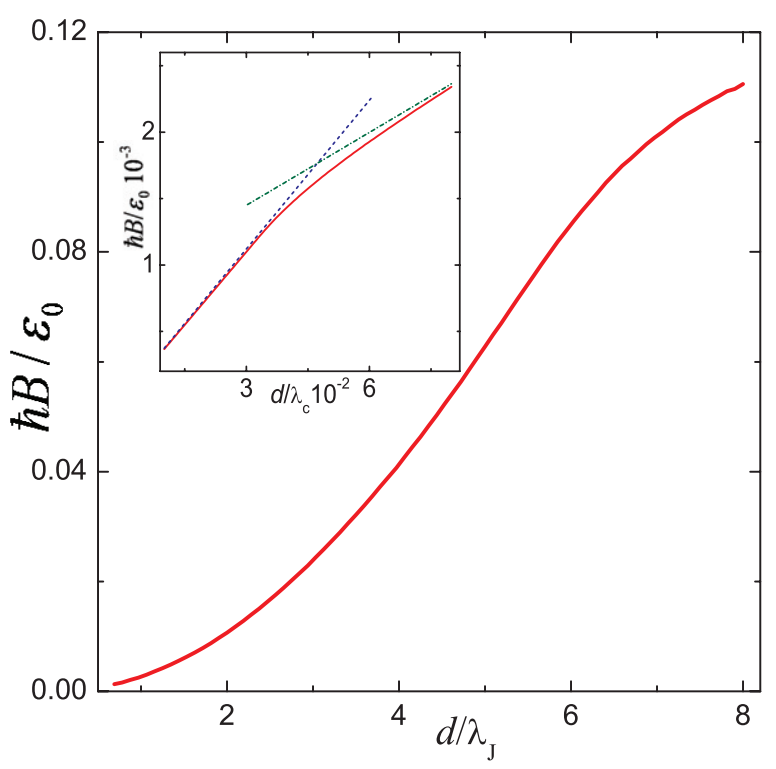

Fig. 3: (Color online) Tunnelling exponent $B$ vs. $d$ for $0-\pi$ junction in the main approximation; $I / I_{c}(d)=0.99$. In the inset: $B$ vs. $d / \lambda_{c}$ at $j=0.99, \gamma s / \lambda_{c}=10^{-2}$ (red solid line) for a stack of JJs. The slope of the asymptote of $B(d)$ at $d \gg d_{\min }$ (green dot-dashed line) is twice smaller than the slope of $B(d)$ at $d<d_{\min }$ (blue dashed line). The value $d_{\min } \approx 0.033 \lambda_{c}$.

The distribution of $\varphi$ is non-uniform in the $0-\pi$ junction even at $I=0$ [11] and a $0-\pi \mathrm{JJ}$ cannot be described within the "particle approximation" for any $d$. The dependence of the tunnelling exponent on $d$ for a $0-\pi$ junction at $I / I_{c}(d)=0.99$ is shown in fig. 3 . The analysis of the eigenvalue problem shows that within the considered range of $d$, there exists only one small parameter $\mu_{0}$ and the function $\psi(0, x)$ corresponds to the antivortex nucleus at the left edge of the junction.

Tunnelling in stacks of junctions. - In layered superconductors described as a stack of JJs of height $D$, a fluxon can tunnel through a certain contact located at $D_{1}$. This process can be described by the non-local Lagrangian

$$
\begin{aligned}
\mathcal{L}= & \frac{\varepsilon_{0}}{2} \int_{0}^{d} \mathrm{~d} x\left[\psi \int_{0}^{d} K\left(x ; x^{\prime}\right) \frac{\partial^{2} \psi}{\partial x^{\prime 2}} \mathrm{~d} x^{\prime}\right. \\
& \left.-\left(\frac{\partial \psi}{\partial \tau}\right)^{2}-\sqrt{1-j^{2}} \psi^{2}+\frac{\psi^{3}}{3}\right],
\end{aligned}
$$

which can be obtained following the approach in ref. [5]. Here $\varepsilon_{0}=i_{0} D \lambda_{c} /\left(4 e \omega_{J}\right), \lambda_{c}$ is the out-of-plane penetration depth,

$$
\begin{aligned}
& K\left(x ; x^{\prime}\right)=(\gamma s / d) \\
& \times\left[\chi_{0}+\sum_{n=1}^{\infty} \chi_{n} \cos \left(k_{n} x\right) \cos \left(k_{n} x^{\prime}\right)\left(1-j^{2}\right)^{1 / 4} / k_{n}\right],
\end{aligned}
$$




$$
\begin{gathered}
\chi_{n}=\cosh \left(\nu_{n} D_{1}\right) \cosh \left[\nu_{n}\left(D-D_{1}\right)\right] / \sinh \left(\nu_{n} D\right), \\
\nu_{n}=\sqrt{k_{n}^{2}\left(1-j^{2}\right)^{-1 / 2}+1},
\end{gathered}
$$

$k_{n}=\pi n \lambda_{c} / d$, anisotropy $\gamma$, and interlayer distance $s$. Note, that there are two different characteristic sizes in the system. The current changes on the scale of $\lambda_{c}$, whereas a characteristic size of the fluxon is $\gamma s \ll \lambda_{c}$. Following the numerical procedure described above and taking into account that the fluxon can tunnel through any junction, we calculate numerically the dependence of $\Gamma$ on the size $d$ of the junction. We also estimate the minimal width of the junctions,

$$
d_{\min }=2 \pi \gamma s / 5\left(1-j^{2}\right)^{1 / 4}
$$

when the "particle" approximation becomes invalid and we should take into account two tunnelling channels. The dependence of the tunnelling exponent $B$ on $d$ is shown in the inset of fig. 3 for $D_{1}=D / 2$. The function $B(d)$ increases with $d$ both at $d<d_{\min }$ and $d>d_{\min }$, but at large $d$ it has a twice smaller slope. Note that recent analytical results [5] provide good semi-quantitative estimates of the tunnelling exponent $B$.

Conclusions. - Here we show that the fluxon tunnels as a particle only through short JJs. For either longer JJs, $0-\pi$ junctions, or JJ's stacks a field-theory approach is required to obtain the correct value of the escape rate, which can be many orders of magnitude higher than the one predicted without taking into account the spatial dependence of the phase difference across the contact. We also demonstrate that the behavior of the MQT escape rate $v s$. the junction length is qualitatively different for usual, $0-\pi$ junctions, and JJ's stacks.

We acknowledge stimulating discussions with Prof. J. KELLER, and also partial support from the NSA, LPS, ARO, NSF grant No. EIA-0130383, JSPS-RFBR 06-02-91200, JSPS CTC program, ESF AQDJJ networkprogramme, RFBR 06-02-16691, MEXT Grant-in-Aid No. 18740224, and the EPSRC via No. EP/D072581/1, $\mathrm{EP} / \mathrm{F} 005482 / 1$.

\section{REFERENCES}

[1] Takagi S., Macroscopic Quantum Tunneling (Cambridge University Press, Cambridge) 2002.

[2] You J. Q. and Nori F., Phys. Today, 58, No. 11 (2005) 42.

[3] Inomata K., Sato S., Nakajima K., Tanaka A., Takano Y., Wang H. B., Nagao M., Hatano H. and Kawabata S., Phys. Rev. Lett., 95 (2005) 107005; Lombardi F., TAFuri F., Barone A., Rotoli G., Delsing P. and Claeson T., Phys. Rev. Lett., 94 (2005) 087003; BAuch T. et al., Science, 311 (2006) 57.

[4] Jin X. Y., Lisenfeld J., Koval Y., Lukashenko A., Ustinov A. V. and Müller P., Phys. Rev. Lett., 96 (2006) 177003.

[5] Savel'ev S., Rakhmanov A. L. and Nori F., Phys. Rev. Lett., 98 (2007) 077002; 269901.

[6] Larkin A. I. and Lee P. A., Phys. Rev. B, 17 (1978) 1596; Kato T. and Imada M., J. Phys. Soc. Jpn., 65 (1996) 2963; Shnirman A., Ben-Jacob E. and Malomed B., Phys. Rev. B, 56 (1997) 14677; Wallraff A. et al., Nature (London), 425 (2003) 155; LI H., SHEN S.-Q., Liang J.-Q. and LiU W.-M., Phys. Rev. B, 72 (2005) 014546; Savel'ev S., Rakhmanov A. L., Hu X., Kasumov A. and Nori F., Phys. Rev. B, 75 (2007) 165417; Goldobin E. et al., Phys. Rev. B, 72 (2005) 054527.

[7] Savel'ev S., Yampol'skit V. and Nori F., Phys. Rev. Lett., 95 (2005) 187002; Physica C, 445-448 (2006) 183; Savel'ev S., Rakhmanov A. L. and Nori F., Phys. Rev. Lett., 94 (2005) 157004; Physica C, 445-448 (2006) 180; Savel'Ev S., Yampol'skit V., Rakhmanov A. and Nori F., Phys. Rev. B, 72 (2005) 144515; Physica C, 437-438 (2006) 281; 445-448 (2006) 175; YAMPOL'SKII V. A., Savel'ev S., Usatenko O. V., Mel'nik S. S., Kusmartsev F. V., Krokhin A. A. and Nori F., Phys. Rev. B, 75 (2007) 014527; SAvel'Ev S., Rakhmanov A., YAmpol'ski V. and Nori F., Nat. Phys., 2 (2006) 521; Savel'ev S., Yampol'skiI V. A., Rakhmanov A. L. and Nori F., Phys. Rev. B, 75 (2007) 184503; Savel'ev S. and Nori F., Nat. Mater., 1 (2002) 179; Tonomura A., Nat. Mater., 5 (2006) 257; Cole D. et al., Nat. Mater., 5 (2006) 305; Cole D. et al., Europhys. Lett., 76 (2006) 1151; Ustinov A. V. et al., Phys. Rev. Lett., 93 (2004) 087001; Goldobin E. et al., Phys. Rev. E, 63 (2001) 031111.

[8] Kawabata S. et al., Phys. Rev. B, 70 (2004) 132505; 72 (2005) 052506.

[9] Coleman S., Phys. Rev. D, 15 (1977) 2929.

[10] Caldeira A. O. and Leggett A. J., Phys. Rev. Lett., 46 (1981) 211.

[11] Hilgenkamp H., Nature, 422 (2003) 50. 